\title{
The RAS Scheduling Algorithm Based on 802.11n WIFI Router
}

\author{
Meng Han ${ }^{1}$,Wenyu Chen ${ }^{* 2}$, Wei $\mathrm{Pi}^{2}$, Wen $\mathrm{Li}^{2}$ and Ru Zeng ${ }^{2}$ \\ ${ }^{1}$ School of Political Science and Public Administration \\ University of Electronic Science \& Technology of China, \\ Chengdu, P.R. China \\ ${ }^{2}$ School of Computer Science and Engineering \\ University of Electronic Science \& Technology of China, \\ Chengdu, P.R. China \\ hmuestc@126.com
}

\begin{abstract}
In order to solve the frame overhead to the influence of frame transmission performance, improve the throughput, lower hybrid scenario user acces delay, improve user (802.11 n) especially the user experience, this paper aimed at different rate of terminal equipment, designs the RAS algorithm, this algorithm through optimizing scheduling, in does not affect user delay at low speed, reduce the high-speed users send time delay.The algorithm is reasonable configuration of channel resources, make full use of the high speed channel rate, rate of various users (especially in high speed rate of users) to improve the user experience. In the Chariot on Atheros9341 chip using TCP service test 802.11 n AP topology network end-to-end delay and throughput, thus the proposed method was verified. The measured results showed that the proposed method can improve the AP client in a high-density environment effective machine capacity, and increase the throughput, reduce the delay.
\end{abstract}

Keywords: wireless networking, frame aggregation, frame scheduling, rate-aware

\section{Introduction}

According to newly released in January 2014, according to the China Internet development report as of December 2013, scale of Chinese Internet users reached 618 million, the scale of mobile Internet users reached 500 million, Internet users of mobile Internet use has risen to $81.0 \%$ [1].The high flow especially the development of mobile applications. According to the report, as of December 2013, our country the numbers of users online watch and download the video phone end of 247 million, the data in 2012 is 135 million, an increase of 112 million, increasing 83.8\%.Mobile video business also increases as mobile Internet's fifth-biggest application. Users surf the web from previous computers to mobile terminal equipment, smart phones and the development of the intelligent mobile terminal has attracted a wider range of people using mobile phones to surf the Internet. These needs directly stimulated WIFI network popularization as well as the improvement of infrastructure, making access to more and more towards the ubiquity of scene change. At present most of the devices connected to the Internet is through wi-fi router access, network performance of the bandwidth allocation in addition to the operator under the influence, the more important it is in this part wi-fi router access and scheduling. It is in this context, optimize the WIFI router's performance is more important.

\footnotetext{
* School of Computer Science and Engineering University of Electronic Science \& Technology of China,Chengdu, P.R. China
} 
In view of the wireless protocol optimization at home and abroad have a lot of related research.For MAC layer mainly focuses on the study of access technology, the research frame polymerization and on the study of the fair scheduling and resource allocation.

Sadeghi et al., presented in [2] OAR (Opportunistic Auto - Rate) algorithm, the algorithm is improved using channel conditions for timing the frame of aggregation. When the physical found at present can be sent at a higher rate, it will notify the MAC layer, MAC layer is in accordance with the basic rate and sends a frame for the same time, aggregated into an aggregate frame, in order to more suitable for the polymerization rate of the physical frame to send.Aimed at the better for channel users are allowed to transmit larger aggregate size of the frame.This makes the transmission rate and increased 802.11 MAC overhead implements a fairness.

Skordoulis et al. In [3] proposed two level frame aggregation mechanisms. The two layers of polymerization mechanism combined the two frames of $802.11 \mathrm{n}$ polymerization way.First, the upper MAC layer will send packets aggregation in A - MSDU way, attach A MAC frame head and inspection and at the same time.Then, connect these A - MSDU into A - MPDU.Each of them A - use MAC delimiter separated between MSDU.This way of aggregate relative to A - MSDU improves the maximum aggregate size, relative to A MPDU lessens the overhead of MAC frame head.Skordoulis et al. The proposed this mechanism allows the use of BlockACK mechanism is applied to A - MSDU frame.The author will this mechanism with $802.11 \mathrm{n}$ standard proposed frame aggregation mechanisms were compared on throughput rate, better than the standard frame polymerization way.

Bianchi is proposed in [4] based on the two-dimensional Markov chain - distributed coordination function analysis model, the model is used to determine the throughput rate of saturated load in WLAN channel.And YuXia Lin et al. [5] in expanded the function of this model, make it can support frame aggregation mechanisms, and puts forward a simple adaptive algorithm for the optimal frame length and frame aggregation mechanisms used in the ideal channel, study and improve the mechanism.

In the standard IEEE802.11 n [6] proposed the LQ (Long Queue) as the default scheduling algorithm.The algorithm will be sent to the same destination of each group choose a set of business frame can achieve maximum aggregate length frame, and then sent after polymerization.Its purpose is to achieve maximum throughput rate and the maximum frame aggregation.However, this approach does not take into account changes over time, the quality of each site access channel, is simply the same rate as the user as a channel to participate in scheduling.Maximum frame length is not in any channel is the optimal choice.And there is no consider moving in the scene.

MRS (Maximum Rate Scheduling) algorithm [7] is users tend to choose the ideal channel, make its peak channel capacity, in order to improve the overall throughput.This kind of greedy algorithm, will cause a lot of time delay and unfair.

802.11 aspects of the delay performance of CSMA/CA, there have been some research.K. Lu, et al., and p. Chatzimisios defined in [8] and [9] and full load condition is deduced from the MAC layer service time. The service time is take the packet at the beginning of the sender to send to transfer work done, regardless of whether the packet is received.But in fact, the data packets in the actual transmission, since the sender in large might try many times to send both failure time is discarded.

In Chen et al. [10] put forward a kind of unsaturated load cases, obtain MAC layer service time method. The method is based on the MAC service time has nothing to do with the state of the buffer queue based on the assumption of. The method using the M/G $/ 1$ model to get the average queuing delay. In order to avoid complexity analysis, the author through simulation to get the MAC service time.

Focused on these analysis and studies on polymerization frame, and according to the channel state changes aggregate frame on the long research, Skordoulis et al. The proposed mechanism of secondary polymerization frame has the very good reference 
value, because it combines A - MPDU and A - the advantages of the MSDU.But it does not take into account the channel change under different rate of terminal state effect on the polymerization. Dionysius Skordoulis et al. The change according to the channel bit error rate and throughput is worth reference frames long way, but also should be the method validation and optimization in the actual scene.MRS algorithm in scheduling in order to obtain high channel overall throughput for some users get more resource scheduling will cause unfair scheduling. Chen et al. Analysis and optimization for delay of the improved, but the method without considering the influencing factors of delay queuing delay, and in a follow-up study, that in some cases is the main component of the end-to-end delay.

At present, the rate of all kinds of mobile terminal coexist, access networks, together with support $802.11 \mathrm{a} / \mathrm{b} / \mathrm{g}$ of traditional equipment, also has support $802.11 \mathrm{n}$ high-speed equipment.Support and 802.11 a $802.11 \mathrm{~g} / \mathrm{b}$ share the band, and the IEEE $802.11 \mathrm{n}$ standard extends the coexistence management to protect its in $11 \mathrm{a}, 11 \mathrm{~b}, 11 \mathrm{~g}$ of transmission equipment.

But the traditional equipment and high-speed devices (e.g., $802.11 \mathrm{n}$ equipment), the rate and the pure high speed equipment scenario rate and ShiYanJun have obvious difference.At the same time, because the rate of $802.11 \mathrm{n}$ standard support adaptive, this means that the transmission rate can differ according to the quality of channel and terminal and adaptive change rate.Hybrid scenario throughput will have different rate change with the terminal, which will result in $802.11 \mathrm{n}$ equipment for poor channel quality.802.11 standard of CSMA/CA mechanism makes all of the sites have a fair opportunity to gain transmission.But from the point of view of wireless spectrum allocation, low speed relative to the high-speed equipment resources it will need more time to complete the frame transmission.In hybrid scenario, TXOP (transfer opportunity) distribution rule $802.11 \mathrm{n}$ high-speed equipment due to the low speed increases the delay of the $802.11 \mathrm{~b} / \mathrm{g}$.Based on this phenomenon, it can be seen that such scheduling, for $802.11 \mathrm{n}$ high-speed device is not fair. Because there is no play to the advantages of its high-speed equipment fast transmission. True. Because of this, this paper proposes a RAS (Rate Aware Scheduling) frame Scheduling algorithm.

\section{The RAS Scheduling Algorithm}

When multi-rate terminal access, the arrival of the low-speed devices will greatly reduce the network overall throughput and transmission delay of high speed equipment, effect on rate of low-speed devices of other equipment the user experience. Wireless AP management resources and data transmission function entity, has decided to send for the received frame or temporary storage function. In order to reduce delay and improve the throughput and ensure the QoS and end user experience, for the AP and redesign the scheduling function.

In this article, we will send the received frame represents rate of equipment is divided into three interval, rate represents the frame rate, low speed device frame rate range:

$0<$ rate $<=$ LOW_THRESH;Medium-speed equipment frame rate range: LOW_THRESH < rate < = MID_THRESH;High-speed equipment frame rate range: MID_THRESH $<$ rate.

\subsection{Algorithm of the Variables and Parameters}

Rate: the frame Rate of the sender device;

LOW_THRESH: low speed rate range for the equipment in the value of the following devices is defined as a low speed; 
MID_THRESH: medium speed rate range for the equipment under the value, and above LOW_THRESH defined as medium-speed equipment, more than the value of equipment frame belongs to the equipment;

Propor_mid: in medium-speed equipment sent round, the current high speed relative to the medium speed equipment delivery ratio value, when sending a high-speed machine frame after adding 1, when sending a device queue frame after minus propor_mid_thresh at a moderate speed.This value is sent with different frame rate dynamic change, based on the value and propor_mid_thresh has dynamic adjustment with high speed rate of equipment frame and medium speed rate frame sending rate purposes;

Propor_low: in low-speed devices to send in the round, the current high speed devices send ratio value relative to the low speed device, when sending a high-speed machine frame after adding 1; When sending a minus propor_low_thresh after low-speed equipment frame, based on the value and propor_low_thresh has dynamic adjustment with high speed rate of equipment frame and low speed rate frame sending rate purposes;

Propor_low_thresh: low speed device to send ratio threshold method, when propor_low does not exceed this value shows that the current low speed equipment frame is not to get too much, temporarily cache, when propor_low more than the value, low speed frame buffer queue of equipment has been cached more frames, low speed device users feel the need to be considered sending a low-speed equipment frame;

Queue_mid_num: medium speed equipment current frame buffer queue number;

Queue_low_mum: low speed device current frame buffer queue number;

Maximum QUEUE_MID_MAX: medium speed device queue, queue length;

Maximum QUEUE_LOW_MAX: low speed device queue, queue length;

Timer, timer, according to the low speed and medium speed device cache queue is the earliest arrival time frame based on arrival time, if the value is more than $\mathrm{M}$, show the frame in the queue waiting time is too long, need to send in time, the trigger to send the queue frame;

M: frame buffer queue waiting for the service time longest

\subsection{The RAS Scheduling Algorithm Design}

Algorithm steps as follows:

1. Wait for frame, when receiving a frame, according to the frame of the frame header information and the associated logo determine its belongs to which rate range, for example, under normal circumstances, the rate of 11 MBPS of $802.11 \mathrm{~b}$ equipment in low speed range, rate of 54 MBPS $802.11 \mathrm{~g}$ equipment for medium speed range.And according to the specific situation will be in addition to high-speed equipment frame interval of other rate, frame rate for the respective range of frame in response to a queue.Queue length respectively by queue_low_length, queue_mid_length logo.

2. According to different rate range of scheduling rules in proportion to send. When the frame reaches, and identify its success rate range, according to the different interval scheduling scheme for scheduling.

3. If the frame is belong to the low speed range equipment, then see if queue_low_length than QUEUE_LOW_MAX, if more than, then sends frames of all of the low speed device queue, and go to the (3), otherwise, check to see if the parameter propor_low than propor_low_thresh, if it is more than propor_low_thresh, will frame the team, and send the team the first frame, if not greater than otherwise, only the frame team don't do any operation, (3). 
If the frame belongs to medium speed equipment range, see if queue_mid_length than QUEUE_MID_MAX, if more than, will the medium-speed equipment all the frames in the queue to send, and go to the (4);Otherwise check propor_mid, if greater than propor_mid_thresh, the frame of entrance, and send the queue for the first frame If the frame belongs to the frame of high-speed equipment range, according to the following four conditions to send:

If propor_low not greater than propor_low_thresh and propor_mid not greater than propor_mid_thresh, directly to the frame and turn to (3);

If propor_low not greater than propor_low_thresh, but propor_mid than propor_mid_thresh, then send the high-speed frame, and removed from the mediumspeed equipment cache queue team first frame to send, go to (3);

If propor_low than propor_low_thresh, but not greater than propor_mid_thresh propor_mid, then send the high-speed frame, and removed from the low speed device cache queue team first frame to send, to the (3);

If propor_low than propor_low_thresh and propor_mid than propor_mid_thresh, then send the high-speed frame, and from the equipment at low speed and medium speed device sends out the team the first frame, cache the queue to (3);

(3) To examine the timer values, if more than $\mathrm{K}$, will be low or medium speed equipment frame buffer queue all together to send, turn to (4), otherwise don't do any operation, go to the (4);

4. Scheduling end and return.

\section{Algorithm Test}

\subsection{Test Environment and Tools}

Use IxCharoit on network time delay, throughput test, the test will be Madwifi original SDK compared with RAS after scheduling algorithm to improve the performance of the test.

Measured environment as shown in figure 1, the test using the AP chip as shown in Figure 2 .

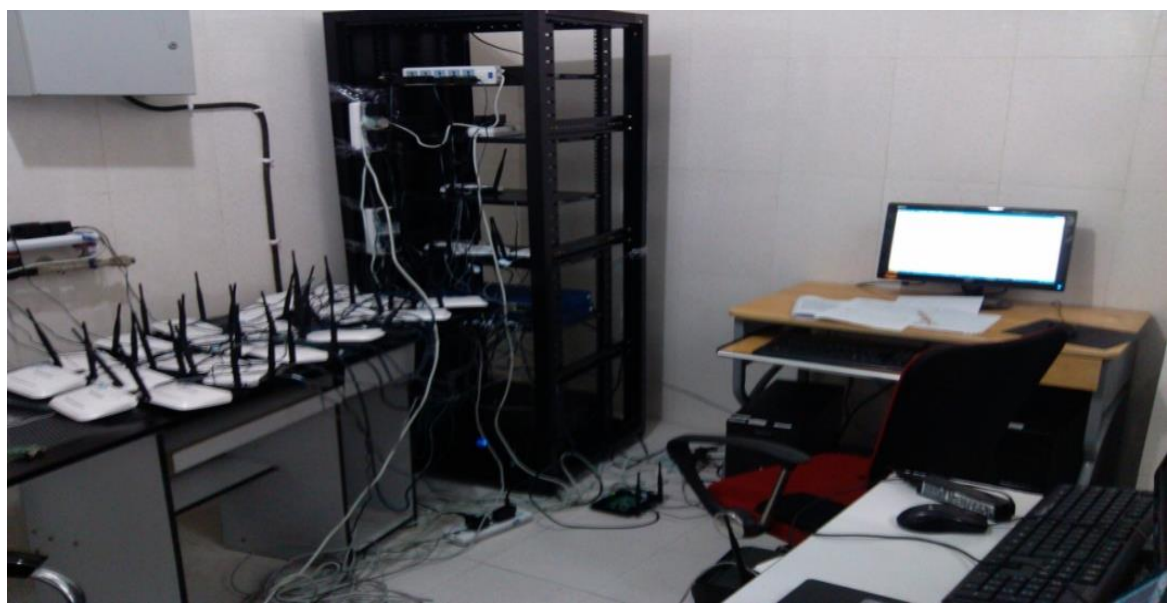

Figure 1. Test Environment 




Figure 2. Test AP Chips

This test will control the deployment on Windows operating system. Will be deployed in the Cent - OS terminal.Control part of the software as its main body function, provides the function of each interface, such as the basic control interface, a design for the entire test and analysis of the interface, is used to test the structure of the script and the choice of interface, according to test results, generate the corresponding test report and displayed in the console, the software also provides the corresponding API interface.In the whole system, as a test execution part, namely the terminal of virtual network card set $1,2, "$ " client, receiving instructions from the control of the system, and then execute the test instruction to test, after will receive the test results and other data back to the control.

\subsection{Test Configuration}

TCP is selected in this test script two-way transmission mode.Each virtual network card to set up 10 TCP traffic, has 5 bar.IP addresses are as follows: the distribution of the IP configuration for 192.168.1.10 IxChariot control end, two virtual LAN server configuration 192.168.1.11 and 192.168.1.12 respectively, the management of virtual LAN server 1 virtual LAN IP address is 192.168.2.1 192.168.2.100, virtual LAN server 1 management of virtual LAN IP address is 192.168.3.1 192.168.3.100.Each IP to end 10 business flow IP using the following configuration, five from 192.168.2.1 to 192.168.3.1 5 data flow and data flow in the opposite direction, other similar.AP end router working mode is configured to "Server", the Client is configured to work patterns "Client".At the same time, in order to test the performance of mixed in scenario, the client is set to different transmission rate.For IxChariot control the configuration, the duration is set to the maximum, to make both ends will be effected according to the need to test.

\subsection{Effective Testing Machine}

Machine amount refers to the limit of the test under the condition of average transport delay and average throughput, $\mathrm{AP}$ can gain access to the maximum number of terminals. The test of average transport delay up to $100 \mathrm{~ms}$, the average throughput is 60 megabits per second.

The Test Case

1. According to the network topology structures, test environment.

2. Activation in a network of 10 client, and connected to the AP. The client USES $802.11 \mathrm{n}$ and AP communication. 
3. Use control side IxChariot to control two clients set (virtual LAN server 1,2), for the AP wireless load with increased 10 client.. After load stability, the average throughput rate not less than 60 megabits per second.

4. Within the scope of the AP wireless access $802.11 \mathrm{n}$ to the client.

5. In monitoring control TCP access test script, lasts for 5 minutes, the average delay in 5 minutes shall be no more than $100 \mathrm{~ms}$.

6. In order to activate the client, and after each activation, repeat observation TCP script and the time delay.

7. When the delay is more than $100 \mathrm{~ms}$ or average throughput rate less than 60 megabits per second, write down the number of clients are activated at this time.

8. Will all equipment power and restart, repeat the above steps, observe every test results, the error should be within 5\%, or to review and configure test environment.

9. Repeat steps (8) twice. Average the result of the test.

\subsection{Effective Quantity of Machine Test Results}

Test results are shown in table 1.Effective quantity of machine is defined as the limit time delay (100 ms) and throughput (60 megabits per second), the number of terminals can access.

Test part of the screenshot as shown in figure 3 and figure 4, see from the results of the test, before his optimized when the number of terminal reach $15 \mathrm{~m}$ or so, throughput peak 80 MBPS, and accordingly the end-to-end delay of more than $100 \mathrm{~ms}$. Practice, the 100 $\mathrm{ms}$ as the delay measure of an application is effective, because when more than the value, the user will feel obvious time delay, especially for voice traffic video, will greatly influence the QoE.

Table 1. Machine Quantity Test Performance Effectively

\begin{tabular}{|c|c|c|c|c|c|c|c|c|c|c|}
\hline \multirow{3}{*}{$\begin{array}{l}\text { Terminal } \\
\text { number }\end{array}$} & \multicolumn{3}{|c|}{ The Average Throughput（Mbps） } & \multicolumn{7}{|c|}{ The Average End-to-End Delay (ms) } \\
\hline & \multirow{2}{*}{$\begin{array}{l}\text { The } \\
\text { Original } \\
\text { SDK }\end{array}$} & \multirow{2}{*}{$\begin{array}{l}\text { Optimized } \\
\text { Version }\end{array}$} & \multirow{2}{*}{$\begin{array}{l}\text { Performance } \\
\text { Optimization }\end{array}$} & \multicolumn{3}{|c|}{ The Original SDK } & \multicolumn{3}{|c|}{ Optimized Version } & \multirow{2}{*}{$\begin{array}{l}\text { Performance } \\
\text { Optimization }\end{array}$} \\
\hline & & & & Average & Max & Min & Average & $\operatorname{Max}$ & Min & \\
\hline 5 & 86.8 & 97.8 & $12.7 \%$ & 35 & 440 & 5 & 24 & 272 & 1 & $31.4 \%$ \\
\hline 10 & 85.2 & 96.6 & $13.4 \%$ & 66 & 490 & 20 & 30 & 368 & 5 & $54.5 \%$ \\
\hline 14 & 80.4 & 92.1 & $14.6 \%$ & 97 & 380 & 23 & 35 & 301 & 3 & $63.9 \%$ \\
\hline 15 & 75.9 & 90.9 & $19.8 \%$ & 100 & 4627 & 27 & 37 & 378 & 8 & $63.0 \%$ \\
\hline 16 & 68.2 & 90.7 & $33.0 \%$ & 103 & 7821 & 66 & 39 & 297 & 9 & $62.1 \%$ \\
\hline 17 & 67.8 & 90.5 & $33.5 \%$ & 117 & 10629 & 44 & 40 & 219 & 9 & $65.8 \%$ \\
\hline 20 & 66.5 & 89.6 & $34.7 \%$ & 143 & 3775 & 77 & 43 & 298 & 12 & $70.0 \%$ \\
\hline 25 & 65.5 & 90.2 & $37.7 \%$ & 165 & 11527 & 30 & 52 & 378 & 20 & $68.5 \%$ \\
\hline
\end{tabular}

Figure 3, shows the SDK effectively after using the RAS machine test, the amount of 25 terminal throughput rate to an average of 90.197 Mbps. Higher than 60 megabits per second test thresholds.

Figure 4, shows under the RAS optimization scheme, effective quantity of machine tests time delay for $52 \mathrm{~ms}$, than $100 \mathrm{~ms}$ of the proposed optimization threshold is further reduced. 




\section{Figure 3. After 25 Terminal Average Throughput was Optimized by Using the RAS 90.197 Mbps}

In this scenario, VOLANS original SDK effective quantity of machine is the 15 units.

After improvement, until 25 terminals, throughput rate remained above 80 MBPS, even the most stable in more than 90 MBPS, while the most obvious even if the end-to-end delay, 25 cases, the terminal time delay is only for $52 \mathrm{~ms}$, much less than the required 100 ms.Is the amount of effective machine is at least $67 \%$.

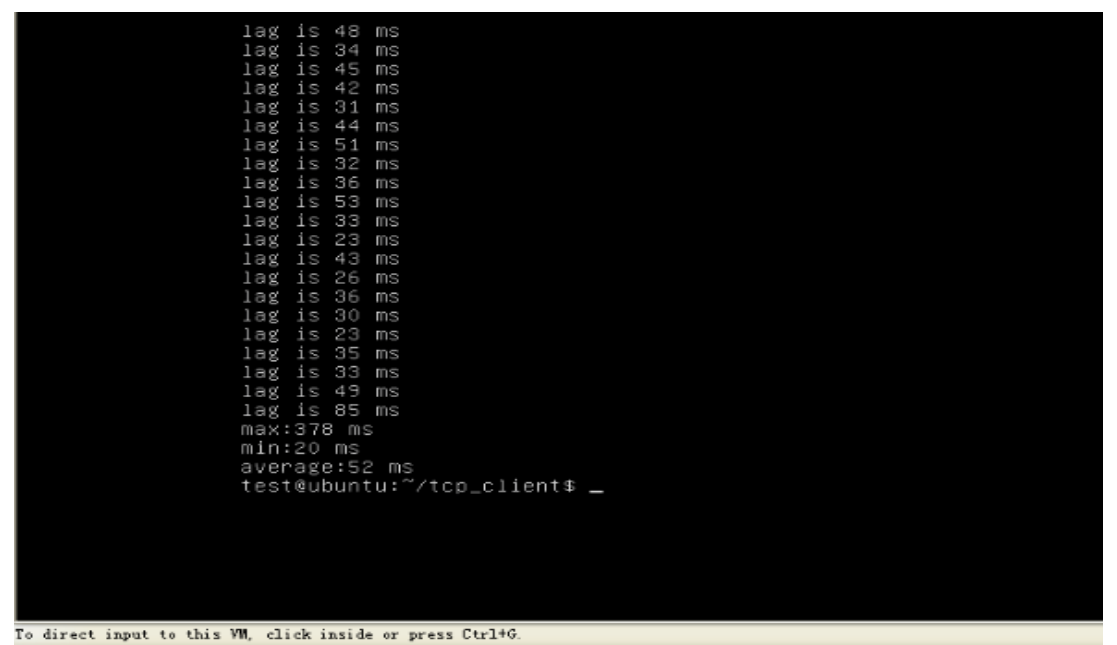

Figure 4. After 25 Terminal was Optimized by Using the RAS Average Delay and MS

From Figure 3, and Figure 4, test results also can be seen that after optimization, the terminal access to the influence that the throughput increase when more is more apparent, when the terminal number is five, throughput increase ratio of $12.7 \%$, and at the time of 15 terminal, improve the performance of $19.8 \%$, when the terminal count to 25 , throughput rate reached $37.7 \%$, thus it can be seen that, after optimization of the RAS and $\mathrm{ABT}$ in high-density environment, performance improvement is more apparent. In addition, for the improvement of time delay, relative to the improvement of the 
throughput rate range is larger, the majority of cases, than up to double the improvement in throughput.

\subsection{Hybrid Terminal Throughput and Delay Test}

To test in a variety of rate of terminal access performance, the complex scene test. Test results are shown in Table 2:

\begin{tabular}{|c|c|c|c|c|c|c|}
\hline \multirow[b]{2}{*}{$\begin{array}{l}\text { Terminal and its } \\
\text { Configuration }\end{array}$} & \multicolumn{3}{|c|}{ The Average Throughput (Mbps) } & \multicolumn{3}{|c|}{$\begin{array}{l}\text { The Average End-to-End Delay } \\
(\mathrm{ms})\end{array}$} \\
\hline & $\begin{array}{c}\text { The } \\
\text { Original } \\
\text { SDK }\end{array}$ & $\begin{array}{l}\text { Optimized } \\
\text { Version }\end{array}$ & $\begin{array}{l}\text { Performance } \\
\text { Optimization }\end{array}$ & $\begin{array}{c}\text { The } \\
\text { Original } \\
\text { SDK }\end{array}$ & $\begin{array}{l}\text { Optimized } \\
\text { Version }\end{array}$ & $\begin{array}{l}\text { Performance } \\
\text { Optimization }\end{array}$ \\
\hline two $(11 \mathrm{n}, 11 \mathrm{~g})$ & 20.7 & 45.4 & $119.3 \%$ & 69 & 23 & $66.7 \%$ \\
\hline two $(11 \mathrm{n}, 11 \mathrm{~b})$ & 18.2 & 49.1 & $169.8 \%$ & 85 & 27 & $68.2 \%$ \\
\hline $\begin{array}{l}\text { fifteen ( seven MCS15 } \\
11 \mathrm{n} \text { 、 three MCS7 } \\
11 \mathrm{n} \text { 、 three } 54 \mathrm{M} \\
11 \mathrm{~g} \text {, two } 5.5 \mathrm{M} 11 \mathrm{~b})\end{array}$ & 21.3 & 42.1 & $97.7 \%$ & 197 & 59 & $70.1 \%$ \\
\hline
\end{tabular}

Table 2. The RAS Optimization Scheme is Different from the Original SDK Access Rate Terminal Performance Contrast Test

The test key screenshots as shown in Figure 5 and Figure6.

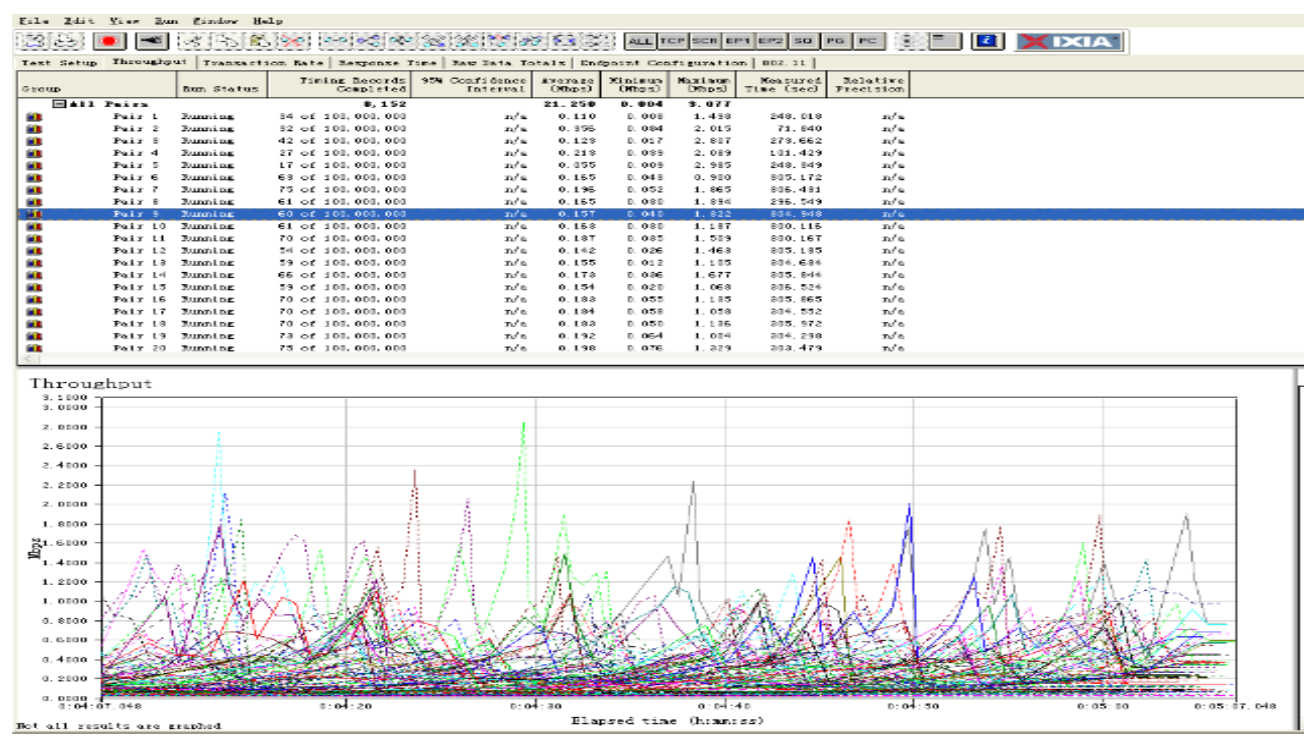

\section{Figure 5. VOLANS Original Edition SDK 15 Terminal Complex Scene Average Throughput Rate 21.259 Mbps}

Figure 5, shows under complex scene, use original SDK test, the average throughput of 21.259 Mbps, its throughput can spontaneously and need to be improved.

See from Figure 6, when using the RAS after optimization of the SDK, average 42.078 Mbps throughput under complex scene, relative to improve before $21.259 \mathrm{Mbps}$, an average of $20.819 \mathrm{Mbps}$ throughput rate increased, and improve the proportion is $97.7 \%$, the optimization effect is obvious. 
In Figure 7, is also the original SDK, test time delay to an average of $197 \mathrm{~ms}$, far more than $100 \mathrm{~ms}$ client feel thresholds.

As can be seen from the mixed in the scenario testing, optimization scheme based on the RAS proportion for the increase of throughput is even more significant, can reach $169.8 \%$.In a complex scenario tests also increased by $97.7 \%$.Throughput lower under the complex scene is a normal phenomenon, because of complex scenes with machine quantity test scenario is contained in different equipment at low speed, in under the condition of low speed device access, the performance of high-speed equipment will be greatly affected.Delay optimization and with those observed in the test machine quantity, proportion of performance improvement can reach $70.1 \%$, the way adopted by the RAS based in actual scene to achieve the goal of good to optimize the network performance.

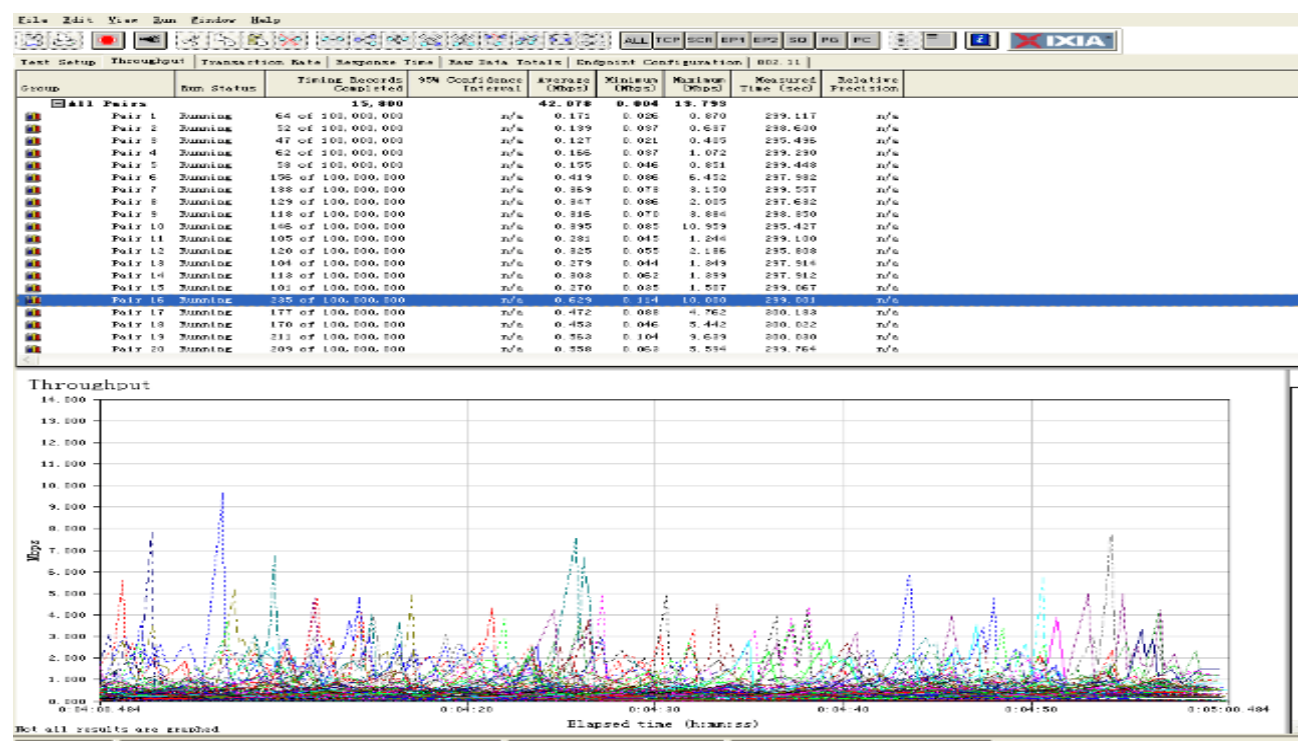

Figure 6. Combinatorial Optimization Version 15 Terminal Complex Scene Average Throughput Rate 42.078 Mbps

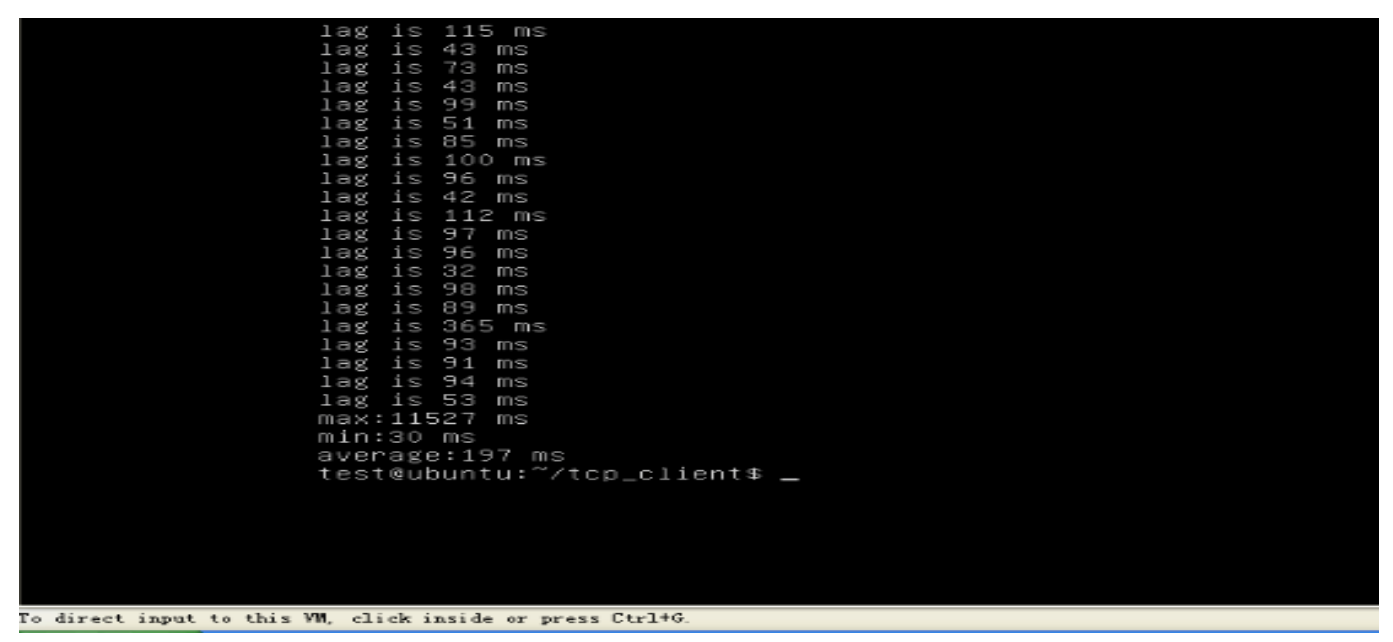

Figure 7. Original Complex Scene SDK15 Terminal an Average of 197 ms Delay 


\subsection{Further Analysis of the Results}

In order to further analysis of the proposed one of the core purpose of the RAS algorithm, namely to the improvement of the user experience, a new definition a test scenario, test topology as mentioned above, still is in control of the client and the client's choice and the configuration change slightly.Introduce three clients are: rate of MCS15 $802.11 \mathrm{n}$ client, rate of $54 \mathrm{Mbps}$ rate of $802.11 \mathrm{~g}$ client and client 5.5 Mbps 802.11 b.Control IxCharoit 5000 packets to each downlink channel.And then test the scenarios each client before, during, and after the introduction of the RAS algorithm in packets take the time.

Typically, the user wants to own the ability of network to ensure the rate of cases within the shortest possible time to complete, the longer the worse experience.Here will be gained by the network users through business service time as a measure of user experience $(\mathrm{QoE})$, under the condition of without affecting the overall rate, shorter service time, the deadline for the earlier, the higher the QoE, on the other hand, is the lower.

Test results show that when using the original deal, the high school and finish time, at an average rate of low speed device users high-speed users in '06' 30 ', get the service time of 06 ' 30 ', 'in the low speed device users are completed in 20', 40 ', get 20 ', 40 ', 'service time.

Compare the above results can be seen, compared with the original plan, the RAS for user QoE optimization effect is obvious, especially for the users, and it is in does not affect the low speed device the user experience, and appropriately raise the medium-speed equipment under the premise of the user experience.

Test results, the users $(802.11 \mathrm{n})$ after the RAS optimization scheme is introduced, the rate of 10.2 Mbps increased to $41.29 \mathrm{Mbps}$, rate did not decline as well as the ascension, and the service time and deadline from 30 '06' 'to' 32 ', '01 shorten, $74.41 \%$ to $74.41 \%$ performance improvement, medium-speed machine increases $61.96 \%$, low speed device did not decline, increased by $0.5 \%$.

\section{Conclusion}

Based on the current MAC frame aggregation and scheduling technology analysis, find out the frame and problems to be solved in polymerization, in view of the actual scenario effective machine volume is low, access equipment at low speed after the characteristics of throughput and delay decline significantly, modify MAC frame send scheduling scheme and the RAS scheduling algorithm is designed. The algorithm by modifying the Madwifi of open source, and run on a $802.11 \mathrm{n}$ wi-fi router (AP). Categorizing mixing rate of different terminals in the scene, and scheduling respectively, give full consideration to the need of high speed equipment, as well as to improve the throughput and reduce the delay effect, both in the low speed device, make them on the basis of the previous user service must be improved. After the measured found that RAS proposed algorithm not only improves the throughput, reduces the time delay, more greatly improve QoE. Optimization process, for frame scheduling objects, consider the coarser, still can continue to further analysis in this aspect and the reasonable classification, not just the root.

\section{References}

[1] China Internet development statistics report [R]. CNNIC, 2014.1:4-10, 23-29,55-89, pp. 98-100.

[2] B. Sadeghi, V. Kanodia, A. Sabharwal, et al., Opportunistic Media Access for Multirate Ad hoc Networks[C], ACM MOBICOM, Atlanta, Georgia, USA .Sept.( 2002).

[3] D. Skordoulis, Q. Ni, H.H. Chen, et al. , "IEEE 802.11n MAC Frame Aggregation Mechanisms for Next-Generation High-Throughput WLANs[C]", IEEE Wireless Communications, vol. 15, no. 1, Feb (2008), pp. 40-47.

[4] G. Bianchi, Performance analysis of the IEEE 802.11 distributed coordination function, Selected Areas in Communications[J], IEEE Journal, vol. 18, no.3, Mar ( 2000) pp. 535-547, 
[5] Y. Lin and V. W.S. Wong, "Frame Aggregation and Optimal Frame Size Adaptation for IEEE 802.11n WLANs[C]", Global Telecommunications Conference, (2006), pp. 1-6.

[6] SA.Mujtaba , "TGn sync proposal technical specification" 3[S]. "TGn Sync Technical Proposal R00", Aug. 13 (2004).

[7] R. Knopp, P.Humblet, "Information capacity and power control in single cell multi-user communications[C]". IEEE International Conference on Communications (ICC) 1995, vol.1, Seattle, USA, June (1995), pp. 331-335.

[8] K. Lu, D. Wu, Y. Fang et al., "Performance analysis of a burst-frame-based MAC protocol for ultrawideband ad hoc networks[J]”, Proc IEEE ICC , vol. 5 ,May (2005), pp. 2937-2941.

[9] P. Chatzimisios, V. Vitsas and A.C. Boucouvalas, Throughput and delay analysis of IEEE 802.11 protocol[J], the IEEE InternationalWorkshop on Networked Appliances, Liverpool ,Oct. (2002), pp. $168-174$.

[10] Y. Chen, Q.-A. Zeng and D.P. Agrawal, "Performance evaluation for IEEE 802.11e enhanced distributed coordinated function[J], "Wireless Communications and Mobile Computing" vol. 4, no. 6, (2004), pp. 639-653. 